\title{
INFECTION
}

\section{Ceftolozane-tazobactam gives a new ASPECT to the fight against antimicrobial resistance}

Ceftolozane-tazobactam is superior to levofloxacin in patients with complicated urinary tract infections (cUTIs) or pyelonephritis, including in patients with resistant uropathogens, according to new research. "Urinary tract infections are an important clinical entity and provide some of the largest reservoirs of resistant and multi-resistant pathogens in the healthcare setting," says Florian Wagenlehner, lead researcher of the Assessment of the Safety Profile and Efficacy of Ceftolozane/ Tazobactam in Complicated Urinary Tract Infections (ASPECT-cUTI) programme. "Antibiotic resistance is sky rocketing, and novel antibiotic substances are urgently needed in the clinic."

The ASPECT-cUTI phase III trial compared ceftolozane-tazobactam, a novel antibacterial with Gram-negative activity, to levofloxacin, an existing firstline antibacterial agent. The study included 1,083 patients with pyuria, pyelonephritis, or cUTIs, who were randomly assigned to receive intravenous infusion of either $1.5 \mathrm{~g}$ ceftolozane-tazobactam every $8 \mathrm{~h}$ or $750 \mathrm{mg}$ levofloxacin once daily, for 7 days. A double-dummy saline infusion was used to mask the treatment groups. Patients with severe renal failure were excluded from the study, owing to uncertainties about the optimum dosing of ceftolozane-tazobactam in these patients.

\section{4 ...novel antibiotic} substances are urgently needed in the clinic $7 y$

"In contrast to previous studies, $\sim 82 \%$ of recruited patients had severe infections, namely pyelonephritis," says Wagenlehner. "Furthermore, baseline susceptibility testing of the two drugs showed that only $2.7 \%$ of Gram-negative pathogens were resistant to ceftolozanetazobactam, whereas $26.7 \%$ were resistant to levofloxacin."
Ceftolozane-tazobactam was superior to levofloxacin in achieving the primary outcome of clinical cure and microbiological eradication. Ceftolozane-tazobactam was also highly effective against multi-resistant and levofloxacin-resistant pathogens.

"These results provide proof of the declining reliability of an empirical levofloxacin therapy in patients with cUTIs," explains Wagenlehner. "Our findings suggest that ceftolozanetazobactam will be a useful addition to the pharmacological armamentarium for the treatment of cUTIs and/or pyelonephritis."

Jessica K. Edwards

This article has also been published in Nat. Rev. Nephrol. (doi:10.1038/nrneph.2015.77)

Original article Wagenlehner, F. M. et al. Ceftolozanetazobactam compared with levofloxacin in the treatment of complicated urinary-tract infections, including pyelonephritis: a randomised, double-blind, phase 3 trial (ASPECT-cUTI). Lancet doi:10.1016/ S0140-6736(14)62482-X 